\title{
BACH ARRAY LINEAR GENERATOR FOR WAVE ENERGY CONVERTERS
}

\author{
Ahmed E. ElGebaly ${ }^{1}$, Mohamed K. El-Nemr ${ }^{2}$ \\ Electrical Power and Machines Engineering \\ Faculty of Engineering, Tanta University \\ Tanta, EGYPT \\ 1ahmed.elgebaly@f-eng.tanta.edu.eg, \\ ²el-nemr@ieee.org
}

\begin{abstract}
linear generators have been proposed as a proper power takeoff system for direct-drive wave energy conversion. Coupled directly to a reciprocating wave energy device, it was declared that linear generators could be a valuable replacement for hydraulic and pneumatic systems. In this paper, the design and performance evaluation of permanent magnet linear generator that implements Halbach array arrangement is established. The designed generator is suitable for direct energy extraction from sea waves at small wave amplitude. The electromagnetic design is performed using finite element method and space harmonic analysis. A comparative study is performed between Halbach array and conventional air-cored linear generators to show the effectiveness of the proposed design. The equivalent circuit based model is utilized to investigate the dynamic performance of the Halbach array generator. The simulated dynamic performance of the designed generator clarifies that the implementation of Halbach array arrangement would improve energy extraction and voltage waveform.
\end{abstract}

Keywords: component; Marine renewable energy, Halbach array, Space harmonic analysis, linear generators, finite element method

\section{Introduction}

The excessive importance of energy sources is emphasized in today's world. Due to the forthcoming enervation of fossil fuels along with their environmental effects, it is vital to develop clean renewable energy sources. Sea wave energy-marine power- is a promising alternative energy recourse. It is the largest renewable energy source on the Earth. It is clean with low running cost. However, is not efficiently implemented yet. Initial surveys show that marine power has the potential of making significant contribution energy market. Specialists estimate that the worldwide economically recoverable wave energy resource is huge. The predicted range is 140 to 750 Trillion $\mathrm{kWh} / \mathrm{year}$ considering that wave-capturing technologies have become fully mature [1].

In the past few decades, various designs have been developed to extract electrical energy from sea waves [2]. The wave energy converters (WECs) can extract the power of ocean or sea waves. They convert wave energy into electricity. These systems either use a water column or buoy body. The buoy body may be on the surface or just below surface [3]. Most of these are progressively moving toward commercial prototype testing. Among these technologies, some devices convert the wave energy into reciprocating motion. Many of these devices create linear low speed reciprocating motion with a peak speed around $2 \mathrm{~m} / \mathrm{s}$. Researchers propose using conventional induction generators to 
provide electrical power. However, that would require a mechanical interface, such as a hydraulic system to transform the low speed reciprocating motion to high-speed rotary motion [4]. Direct-drive electrical power takeoff systems implementing linear generators have the benefit of much simpler construction. A pilot linear permanent magnet (PM) synchronous generator has been designed and constructed for a wave energy converter, which depends on the Archimedes Wave Swing concept [5]. Such linear generators have the disadvantage of high cogging force. The Permanent magnet air-cored tubular linear generator has solved the cogging force problem [6]. An important advantage is that machine can be constructed without the use of magnetic material other than the permanent magnets, eliminating iron parts from the stator and forcer, with their corresponding core loss. That provides more uniform filed, which improves performance. Furthermore, such generators are inherently lightweight [7]. Therefore, in this paper field enhanced further using Halbach arrangement as will be described in the following sections

\section{Halbach array linear generator prototype for WEC}

The performance quality of air-cored tubular permanent magnet synchronous generator depends on the uniformity of the field. Halbach array arrangement can be utilized to produce a uniform

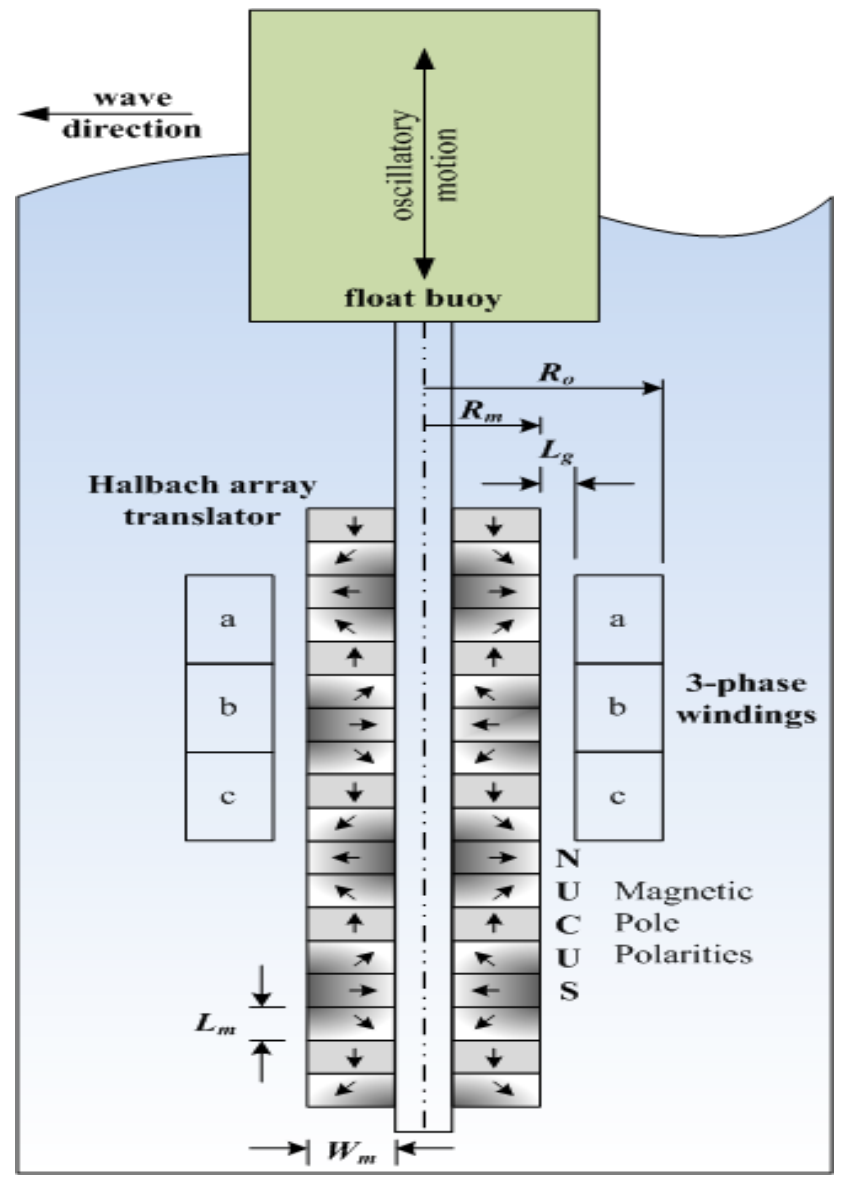

Fig. 1: Halbach array linear generator prototype for WEC magnetic field. The uniform field distribution induces more sinusoidal voltage waveform using this PM arrangement [7]. Since the field is uniform, the machine design is no longer constrained by the air gap size. The field uniformity results in the extraction of very high power peaks over shorter time scales.

Fig.1 illustrates the construction of air cored tubular PM linear synchronous generator with Halbach array arrangement for WEC. The simple design uses a floating buoy directly connected to a PM linear generator. The generator is robustly joined to the bottom of the sea, directly by using wires. The power takeoff system works upon the difference in height between the wave top and wave bottom. Its main advantage is that it is mechanically simple, having only a few moving parts. As shown in Fig.1, Halbach array linear generator has stationary copper coils surround a magnetized tubular translator. The translator consists of a series of oriented polarization permanent magnets such as neodymium-iron-boron. Fields of the magnets are oriented alternatively North (N) and South (S). Surface poles with some magnets to complete the flux path $(\mathrm{C})$. There are some magnets $(\mathrm{U})$ oriented with 45 degrees or 135 degrees to produce uniform flux distribution. The resulting field follows the movement of the translator, cutting the three phase coils separated by 120 degrees electrical. 
To extract power at the low velocities found in WECs, large reactive forces are required, hence large electrical machines. In such machine, the strong magnetic field results in a significant force of attraction between the translator and stator [5]. This should be resisted by the mechanical construction and lubrication system. Significant structural savings can be made if the magnetic forces can be reduced or even eliminated. That can be achieved by constructing a stator with no iron. These features are established via utilizing the Halbach array. The proposed prototype has the following ratings: rated power of $600 \mathrm{~W}$, rated phase voltage of $15 \mathrm{~V}$, frequency of $3 \mathrm{rad} / \mathrm{s}$ and the peak-to-peak wave height of $1 \mathrm{~m}$. The wave parameters are the average values around South Mediterranean Shores [8].

To absorb a proper amount of energy from wave, the proposed prototype may be used with a large number of the generation unit connected in series or parallel. The usage of several units with a small unit rating is suitable for small wave height such as the Mediterranean Sea. The finite element method (FEM) enables to provide the design parameters of the Halbach array linear generator. As shown in Fig.1, the required dimensions are:

$$
\begin{array}{ll}
R_{o}: \text { the generator outer radius }(\mathrm{m}) & L_{m}: \text { PM segment thickness }(\mathrm{m}) \\
W_{p}: \text { the pole pitch }(\mathrm{m}) & W_{m}: \text { PM height }(\mathrm{m}) \\
L_{g}: \text { air gap length }(\mathrm{m}) & R_{m}: \text { the outer diameter of the translator }
\end{array}
$$

The other design parameters are type of PM, number of each phase coil turns, the diameter of the wire and the filling factor of each coil. Numerical study based on FEM analysis to obtain the design parameters provides the required prototype.

\section{Design of WEC generator usingFEM}

To analyze the proposed model, finite-element calculation with the package FEMM is used. Despite of the movement of the actuator, this problem is solved as magnetostatic problem due to the slow motion of the actuator and the low frequency of coil currents also. The electromagnetic field problem can be solved using FEM, at each position. The relation between the vector magnetic potential and current density is described by:

$$
-\frac{1}{\mu} \nabla^{2} \mathbf{A}=\mathbf{J}
$$

where, $(\mu)$ is the permeability which is a function of the flux density for nonlinear materials, $\mathbf{A}$ is the magnetic vector potential and $\mathbf{J}$ is the current density. The advantage of using the vector potential formulation is that all the conditions to be satisfied have been combined into a single equation. Determining A, the magnetic flux density $\mathbf{B}$ is determined from the following equation:

$$
\mathbf{B}=\nabla \times \mathbf{A}
$$

Hereby, the flux linkage $(\lambda)$ of each coil can be obtained at any axial displacement $(x)$ between coil MMF and the PM's MMF. By Faraday's law, the induced voltage in each coil is given by:

$$
e_{a}=\frac{d \lambda_{a}}{d t}=\frac{d \lambda_{a}}{d x} \cdot \frac{d x}{d t}=\frac{d \lambda_{a}}{d x} v_{x}
$$

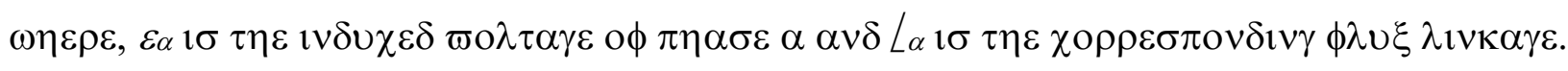


Fig. 2(a) illustrates the flux distribution of the proposed prototype resulting from FEM. The flux is oriented outer the PM cylinder and partially canceled in the inner side of the cylinder. On the other hand, Fig. 2(b) shows the conventional air-cored linear generator where PM segments are imposed between iron segments and each PM segment has orientation reversed to the adjacent one. The FEM analysis is performed using the two-dimensional axisymmetric model. The FEM results illustrate that the Halbach array configuration does not need back iron such as air-cored.The aircored type provides high flux density in the back iron, but the Halbach arrangement avoids this field concentration by the orientation of the field to the out of the cylinder PM. Fig. 3(a) shows the values of radial flux density provided by Halbach design and Fig. 3(b) delivers the values of radial flux density provided by air-cored. According to Fig. 3, the Halbach array develops more radial flux density for the same dimension of air-cored one. Fig. 4 provides the space harmonic analysis of the developed radial flux density for each generator configuration. The values of radial fluxes are determined at the inner surface of three phase windings. The results prove that the Halbach array design provides higher fundamental component and lower harmonics. The higher value of fundamental flux density and lower value of harmonics maximize the value of induced EMF for each phase. According to the previous results, the Halbach array linear generator has better than aircored generator in case of having the same generators volume.

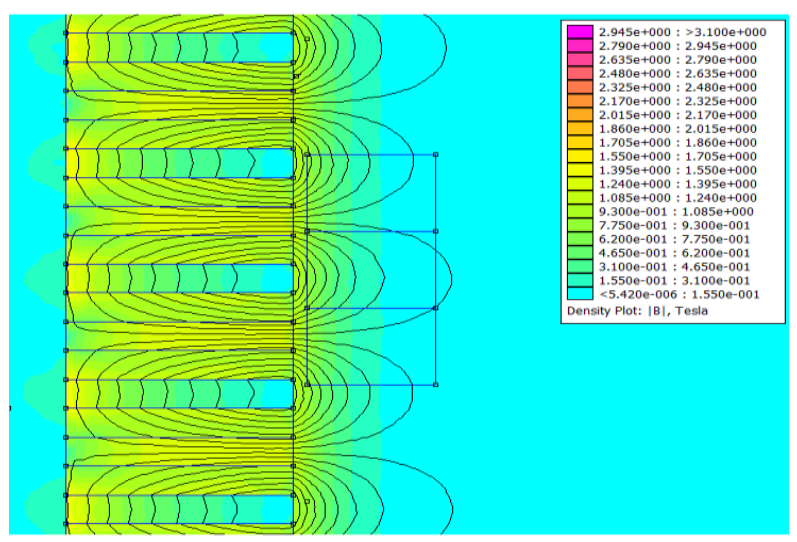

( $\alpha) H \alpha \lambda \beta \alpha \chi \eta \alpha \rho \rho \alpha \psi$

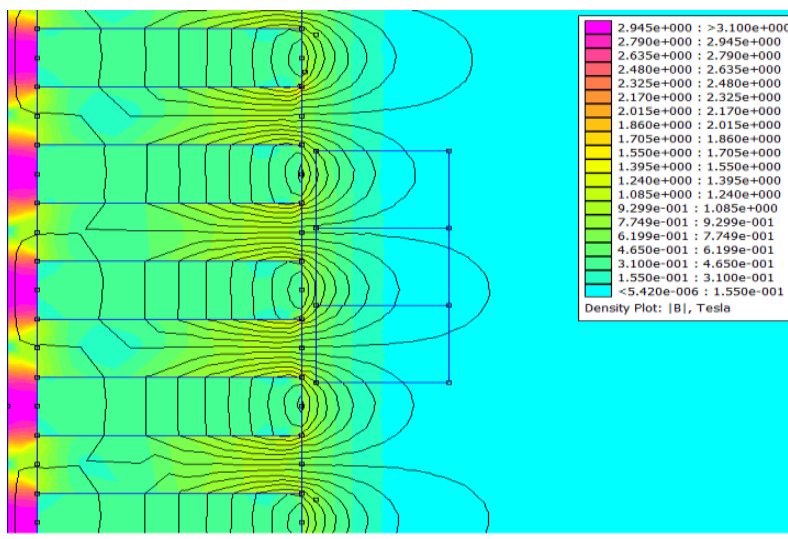

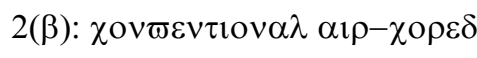

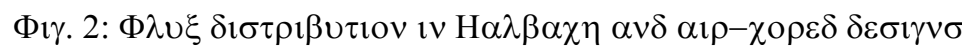

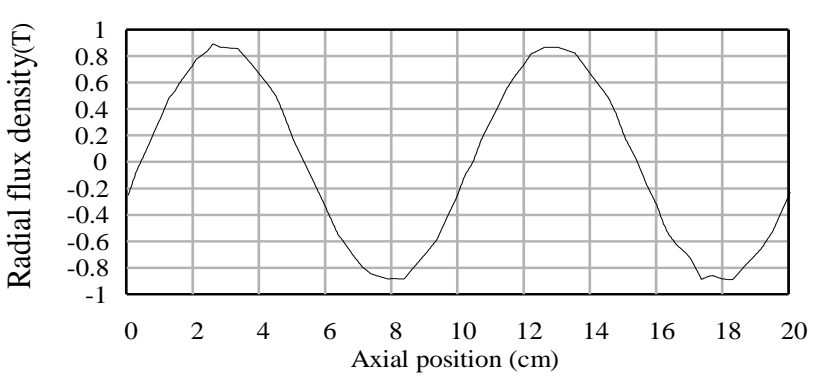

(a) Radial flux density along position due to Halbach array

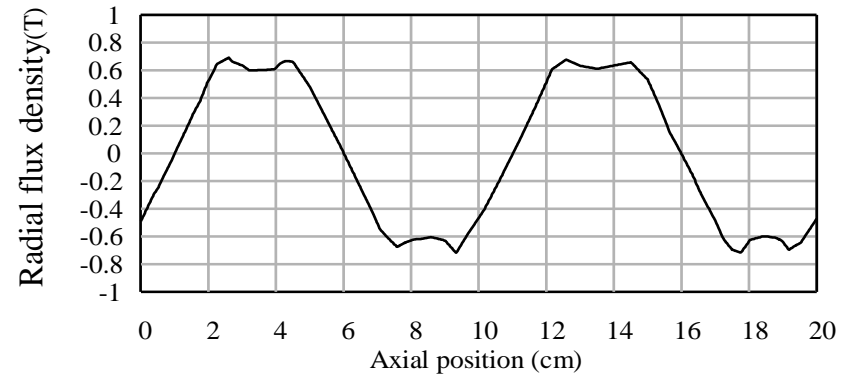

(b) Radial flux density along position due to PMs in air-cored type

Fig. 3 Radial flux density along position in Halbach and air-cored 


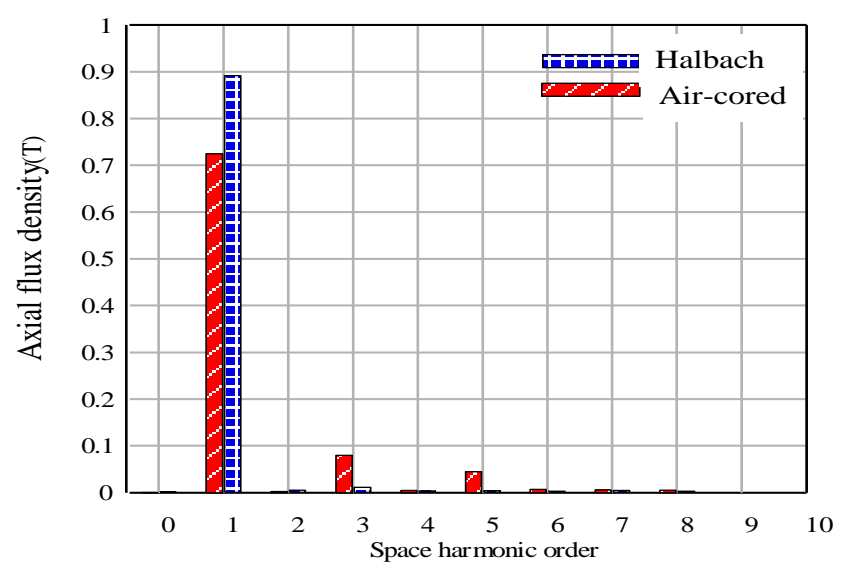

Fig. 4 space harmonic spectrum for developed flux density from two generators

In the proposed Halbach array generator, the rear earth $\mathrm{PM}(\mathrm{NdFeB} 40)$ is used because it has a high coercivity that leads to the small volume generator. Along $1 \mathrm{~m}, 10$ pole pair is arranged. To produce the rated voltage, each phase coil has 250 turns. Fig. 5 shows the flux linkage of each phase along one pole pitch of $0.05 \mathrm{~m}$. The Halbach array arrangement enables to obtain an almost sinusoidal variation of flux linkage over displacement specially the using of 45 degrees or 135 degrees oriented magnet segments. The dimensions have been iteratively examined using the FEMM package to achieve the required performance.

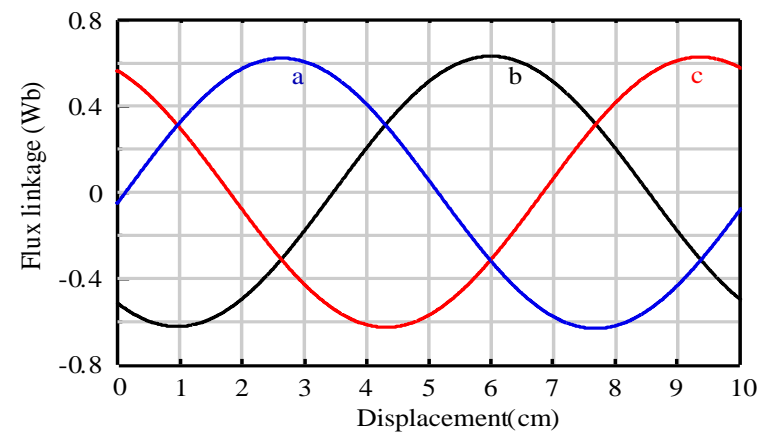

Fig.5 Flux linkage variation with displacement

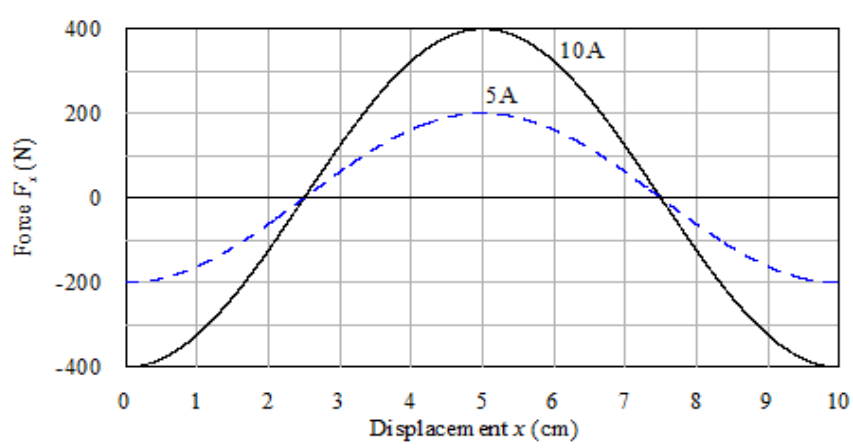

Fig. 6: Axial force verses position for constant current

Table 1 provides the design sheet of the prototype. The internal resistance of each phase equals $1.011 \Omega$ and each coil has self-inductance of $17.6 \mathrm{mH}$. The filling factor of the coil equals $48.17 \%$, which is the proper percentage of this type of generators [4]. The FEMM program can be used to produce the constant current force profile as the relative coil and magnet position changes, as shown in Fig. 6. For two constant currents of 5A and 10A, the force profile is developed. The two maxima have marginally different values, implying the magnitude of the force is dependent on the direction of current with respect to the flux of the magnets. Flux driven by the current can either fortify or oppose the magnetic field of the stator and the force varies.

The presence of coil supported between some coils in the proposed prototype leads to a slightly unbalanced three-phase system. Therefore, it is not probable to make the normal assumptions related to three phase balanced systems, namely that all mutual inductances are equal and the instantaneous sum of the currents is zero. So, the three phase equivalent circuit may be modeled as three EMF sources $e_{a}, e_{b}$ and $e_{c}$. Each is connected in series with an internal resistance $r_{i n}$, selfinductances, two dependent voltage sources to represent the mutual flux linkage with the other phases and the phase load resistance $R_{L}$. A neutral resistance $r_{n}$ is common between all phases. Fig. 7 illustrates the entire three phase equivalent circuit. Hence, the voltage equations are given by (4), (5), (6) and (7). 
Table 1: The dimensions of the proposed prototype

\begin{tabular}{|cc|c|c|}
\hline \multicolumn{1}{|l|}{ Variable } & Value & Unit \\
\hline The generator outer radius & $R_{o}$ & 0.150 & Meter \\
\hline The pole pitch (m) & $W_{p}$ & 0.050 & Meter \\
\hline Air gap length & $L_{g}$ & 0.005 & Meter \\
\hline PM segment thickness & $L_{m}$ & 0.125 & Meter \\
\hline PM height & $W$ & 0.080 & Meter \\
\hline Outer diameter of the translator & $R_{m}$ & 0.100 & Meter \\
\hline Conductor diameter & & 2.0 & Millimeter \\
\hline Number of pole pairs & & 10 & Pole pairs \\
\hline Number of phase turns & & 250 & Turn \\
\hline Winding filling factor & & & \\
\hline
\end{tabular}

IV.Performance of Halbach array linear generator for WEC

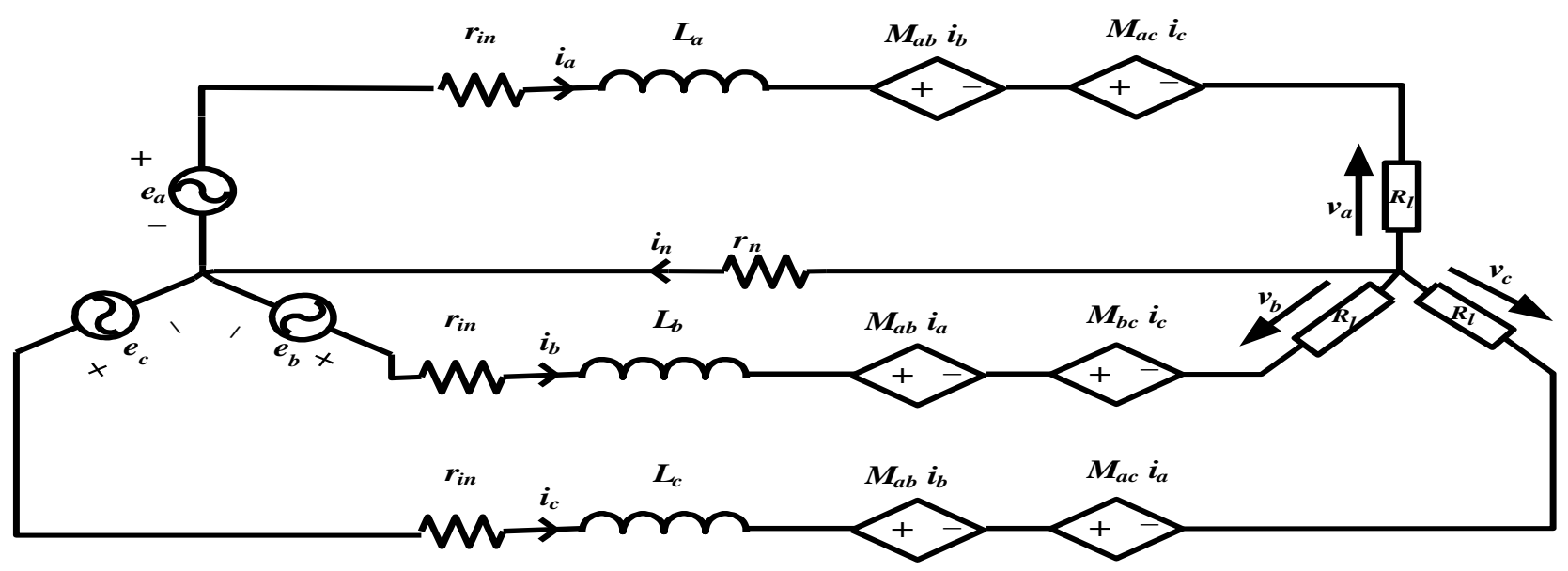

Fig. 7: Three-phase equivalent circuit Halbach array linear generator 


$$
\begin{gathered}
e_{a}=i_{a}\left(r_{i n}+R_{L}\right)+L_{a} \frac{d i_{a}}{d t} \pm M_{c a} \frac{d i_{c}}{d t} \pm M_{a b} \frac{d i_{b}}{d t}+i_{n} r_{n} \\
e_{b}=i_{b}\left(r_{i n}+R_{L}\right)+L_{b} \frac{d i_{b}}{d t} \pm M_{a b} \frac{d i_{a}}{d t} \pm M_{b c} \frac{d i_{c}}{d t}+i_{n} r_{n} \\
e_{c}=i_{c}\left(r_{i n}+R_{L}\right)+L_{c} \frac{d i_{c}}{d t} \pm M_{b c} \frac{d i_{b}}{d t} \pm M_{c a} \frac{d i_{a}}{d t}+i_{n} r_{n} \\
i_{n}=i_{a}+i_{b}+i_{c}
\end{gathered}
$$

where, induced voltages are determined by equation (3) and $r_{n}$ is the neutral resistance. In (3), voltage mainly depends on the velocity of the PM translator with respect to stator phases. The proposed prototype enables to extract energy from both rising and falling waves. To validate the enhanced performance of WEC implementing Halbach array arrangement, the performance of this generator is compared with conventional air-cored generator with the same number of phases [4]. In addition, they have the same number of turns per phase, same PM material and translator height. The comparison takes place at the same input conditions which represents common wave parameters of Mediterranean sea i.e. $1 \mathrm{~m}$ wave height and 1.5 maximum velocity[8]. Fig. 8, 9(a) illustrates the velocity that produced on the floating buoy for both generators. This velocity is assumed to be a sine wave where the positive part means the direction to up and the positive means the opposite direction. The motion of a linear machine is bidirectional and so experiences both positive and negative forces [9].

The difference between air-cored generator and Halbach generator is illustrated according to the following study where the same wave velocity is applied on the two generator models. If the translator is translated, the flux linkage of Halbach generator has sinusoidal waveform with displacement having maximum value of $0.62 \mathrm{~Wb}$ as shown in Fig. 5. On the other hand, normal aircored generator has approximately trapezoidal waveform with displacement with maximum value of 0.52Wb [4]. Fig. 8(b) illustrates the EMF from Halbach generator, but Fig. 9(b) shows the air-cored EMF profile. From the EMF profiles of a linear generator in Fig. 8(b) and Fig. 9(b), it is apparent that phase reversal occurs at the frequency of oscillation, each time the translator reaches its maximum excursion, stops, and changes direction. Fig. 8(b) and Fig. 9(b) illustrate this point at $5.25 \mathrm{~s}$. The induced voltages reach its maximum values at the times the translator makes its maximum positive or negative velocity. As shown in Fig. 8(b) and Fig. 9(b), the EMF values of Halbach generator are larger than the corresponding values of air-cored generator at the same velocity.

To allow for maximum power transfer the load resistance equals the internal phase resistance of $1 \Omega$. Fig. 8(c) and Fig. 9(c) show the load terminal voltage for the two generators. Fig. 8(c) and Fig. 9(c) illustrate the unbalanced terminal three phase voltages. This is due to the unbalance of mutual inductances between phases. At this load, the RMS load current equals 14A and the RMS load voltage is $14 \mathrm{~V}$ for Halbach generator. But for air-cored, the RMS load current equals $11 \mathrm{~A}$ and the RMS load voltage is $11 \mathrm{~V}$. The self and mutual inductances in air-cored generator have larger values than that of Halbach generator due to the using of iron in the first generator. The larger inductances represent an additional reason for low terminal voltage at high loads in air-cored generator. 


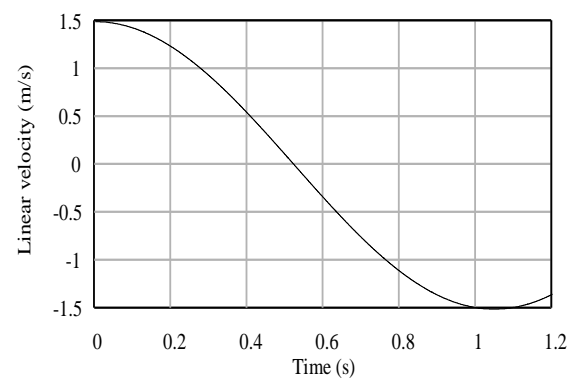

(a) Linear velocity of the linear generator translator

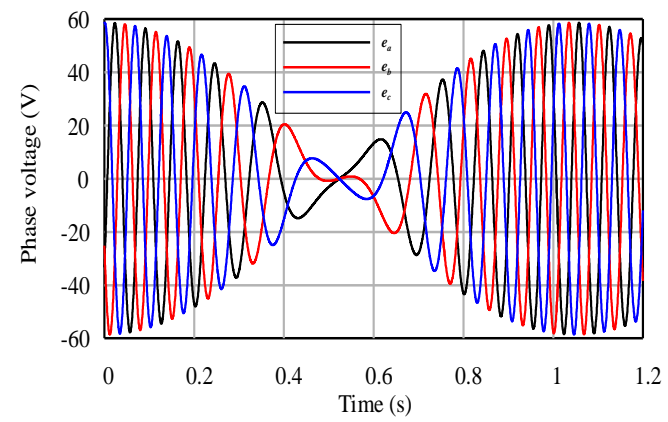

(b) Phases no load voltages

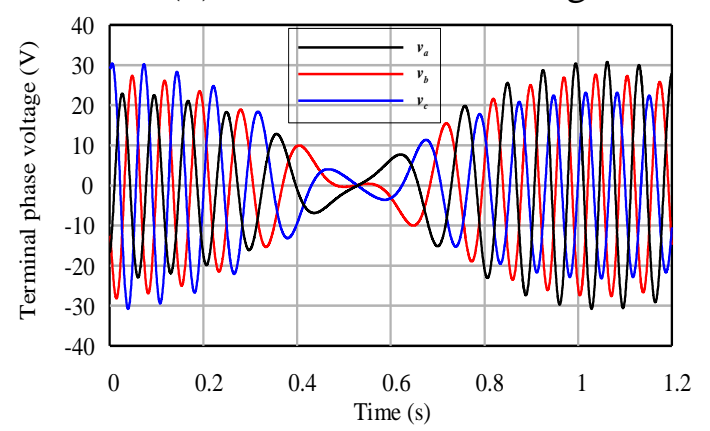

(c) Phase terminal voltages

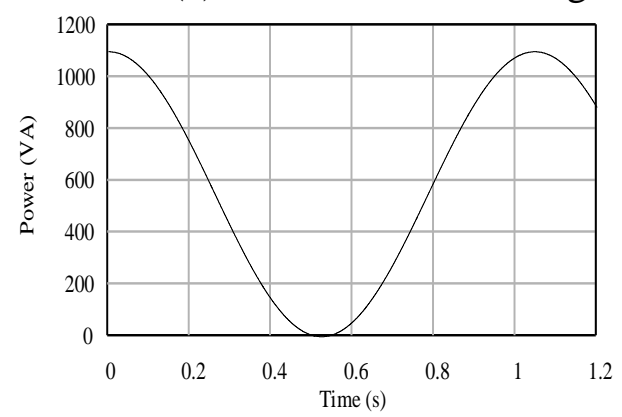

(d) Instantaneous load power

Fig. 8:Performance of Halbach array linear generator for WECs

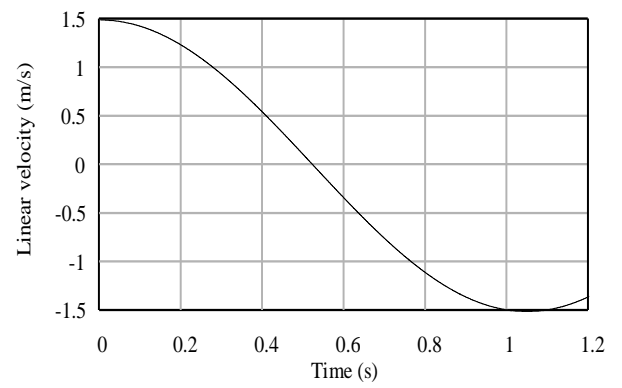

(a) Linear velocity of the linear generator translator

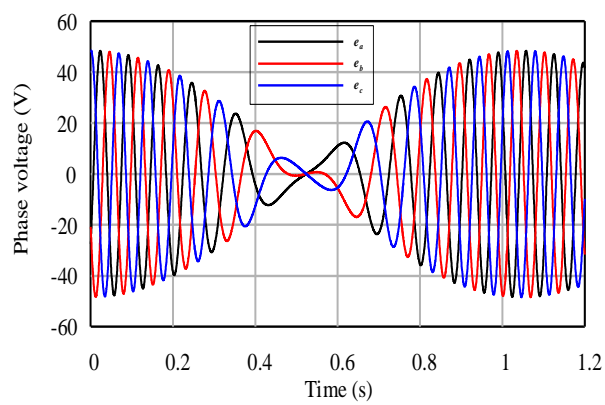

(b) Phases no load voltages

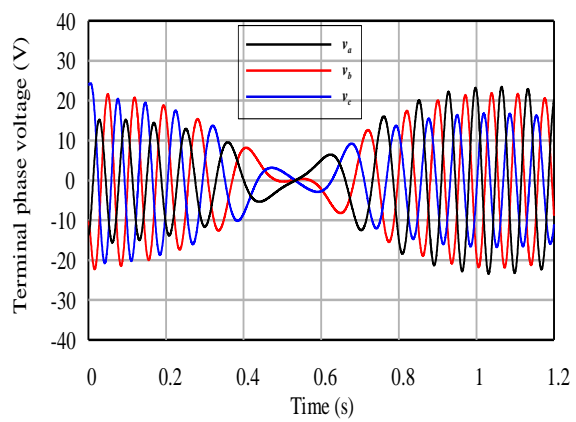

(c) Phase terminal voltages

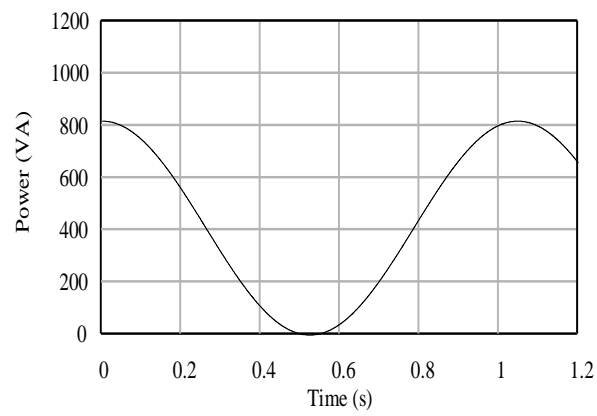

(d) Instantaneous load power

Fig. 9: Performance of Air-cored linear generator for WECs

The total instantaneous power can be calculated according the following equation:

$$
p(t)=i_{a} v_{a}+i_{b} v_{b}+i_{c} v_{c}
$$


where, $i_{a}, i_{b}, i_{c}$ are the instantaneous phase currents and $v_{a}, v_{b}, v_{c}$ are the instantaneous phase voltages. Fig. 8(d) and Fig. 9(d) illustrate the instantaneous load power for the two studied generators. This power takes sinusoidal shape according to the velocity, in other words the extracted power is maximum at the maximum positive or negative velocity. The average developed power equals $550 \mathrm{~W}$ for Halbach array design. In contrary, the air-cored average power equals $410 \mathrm{~W}$.

The results show that Halbach generator is superior to conventional one due to less harmonic contents and more energy density per unit volume. Hereby, power electronics techniques will be implemented to modify the extracted power into electrical grid requirements. These techniques will be characterized by simple construction due to less harmonic content in the output generator voltage. The optimization of the proposed design in combined with the power electronics and mechanical systems represents another required further work before getting a commercial version proper to work in the marine environment.

\section{Conclusion}

The concept of using Halbach array arrangement for WEC linear generator has been introduced. A prototype is designed using FEM technique. The rated output of designed generator is $600 \mathrm{~W}$. That rating is suitable for shores with relatively small wave's amplitude. An equivalent circuit is presented and utilized to evaluate the generator performance. To validate the effectiveness of using Halbach array arrangement, a comparative study has been performed between two similar generators with similar configurations one with Halbach arrangement and the other is just air-cored. The comparative study includes space harmonic analysis and performance at no-load and loading. The results show the proposed generator design has improved energy extraction, less voltage distortion with higher voltage peaks. It is explicitly presented that the proposed arrangement improves the performance. The concept and result would be a valuable for further development of wave energy converters utilizing linear generators.

\section{References}

[1] R. Vermaak and M. J. Kamper, "Design Aspect of a Novel Topology Air-Cored Permanent Magnet Linear Generator for Direct Drive Wave Energy Converters," IEEE Trans. Ind. Electron, vol. 59, no. 5, pp. 2104-2115 May 2012.

[2] M. A. Mueller, N. J. Baker, P. R. Brooking and J. Xiang, "Low Speed Linear Electrical Generators for Renewable Energy Applications," in Proc. Linear Drives in Industrial Applications conference, Birmingham (UK), 2003, pp. 121-124.

[3] R Bedard, G. Hgerman, O. Siddique and et al, "Offshore Wave Power Feasibility Demonstration Project, Project Definition Study, Final Summary Report," Electric Power Research Institute Inc., Palo Alto (USA), Tech. Rep., 2005.

[4] N. J. Baker, M. A. Mueller, and E. Spooner, "Permanent Magnet Air-Cored Tubular Linear Generator for Marine Energy Converters," Proc. Second IEE International Conference on Power Electronics, Machines and Drives, January 2004, pp. 862-867

[5] H. Polinder, B. C. Mecrow, A. G. Jack, P. G. Dickinson, and M. A. Mueller. "Conventional and TFPM Linear Generators for Direct-Drive Wave Energy Conversion,” IEEE Trans. Energy Convers, vol. 20, no. 2, June 2005 
[6] L. Yan1, L. Zhang1, T. Wang1, Z. Jiao1,C. Chen, and I. Chen, "Magnetic Field of Tubular Linear Machines With Dual Halbach Array,’Progress in Electromagnetics Reasearch, vol. 136, pp. 283-299, 2013.

[7] A. Elgebaly, M. El-nemr, M. ElKhazendar and E. Rashad, "Design of Halbach array linear motor actuator for the total artificial heart," inProc. MEPCON'12, Alexandria, Egypt ,2012.

[8] A.M. Abdallah, S.H. Sharaf EL-Din and S.M. Shereet. "Analysis of Wave Observation and Wave Transformation in Abu-Qir Bay, Egypt”, Egyptian Journal of Aquatic Research, vol. 32, no. $1,2006$.

[9] G. Li, G. Weiss,M. A. Mueller ,S. Townley, M. R. Belmont "Wave energy converter control by wave prediction and dynamic programming", ELSEVIER Renewable Energy, vol. 48, pp. 392-403 December 2012. 